\title{
PacBio and Illumina RNA sequencing identify alternative splicing events in response to cold stress in two poplar species
}

\author{
Jingli Yang ${ }^{1}$, Wanqiu Lv ${ }^{1}$, Minzhen Zeng ${ }^{1}$, Yanrui $\mathrm{Fu}^{1}$, and Chenghao $\mathrm{Li}^{1}$ \\ ${ }^{1}$ Northeast Forestry University
}

November 28, 2020

\begin{abstract}
In eukaryotes, alternative splicing (AS) is a crucial regulatory mechanism that modulates mRNA diversity and stability. The contribution of AS to stress are known in many species related to stress. But the post-transcriptional mechanism in poplar under cold stress is still unclear. Recent studies have utilized the advantages of Single Molecular Real Time (SMRT) sequencing technology from Pacific Bioscience (PacBio) to identify full-length transcripts. We, therefore, used a combination of singlemolecule long-read sequencing and Illumina RNA sequencing (RNA-Seq) for a global analysis of AS in two poplar species (Populus trichocarpa and P. ussuriensis) under cold stress. We further identified 1261 AS events in P. trichocarpa and 2101 in P. ussuriensis, among which intron retention, with a frequency of more than $30 \%$, was the most prominent type under cold stress. RNA-Seq data analysis and annotation revealed the importance of calcium, abscisic acid, and reactive oxygen species signaling in cold stress response. Besides, the low temperature rapidly induced multiple splicing factors, transcription factors, and differentially expressed genes through AS. In P. ussuriensis, there was a rapid occurrence of AS events. This study provides new insight into the complexity and regulation of AS during cold stress response in two poplar species.
\end{abstract}

\section{Hosted file}

2859-08-0910-02_Manuscript.pdf available at https://authorea.com/users/379592/articles/ 495772-pacbio-and-illumina-rna-sequencing-identify-alternative-splicing-events-inresponse-to-cold-stress-in-two-poplar-species 


\section{P. ussurensis P. triphocarpa}

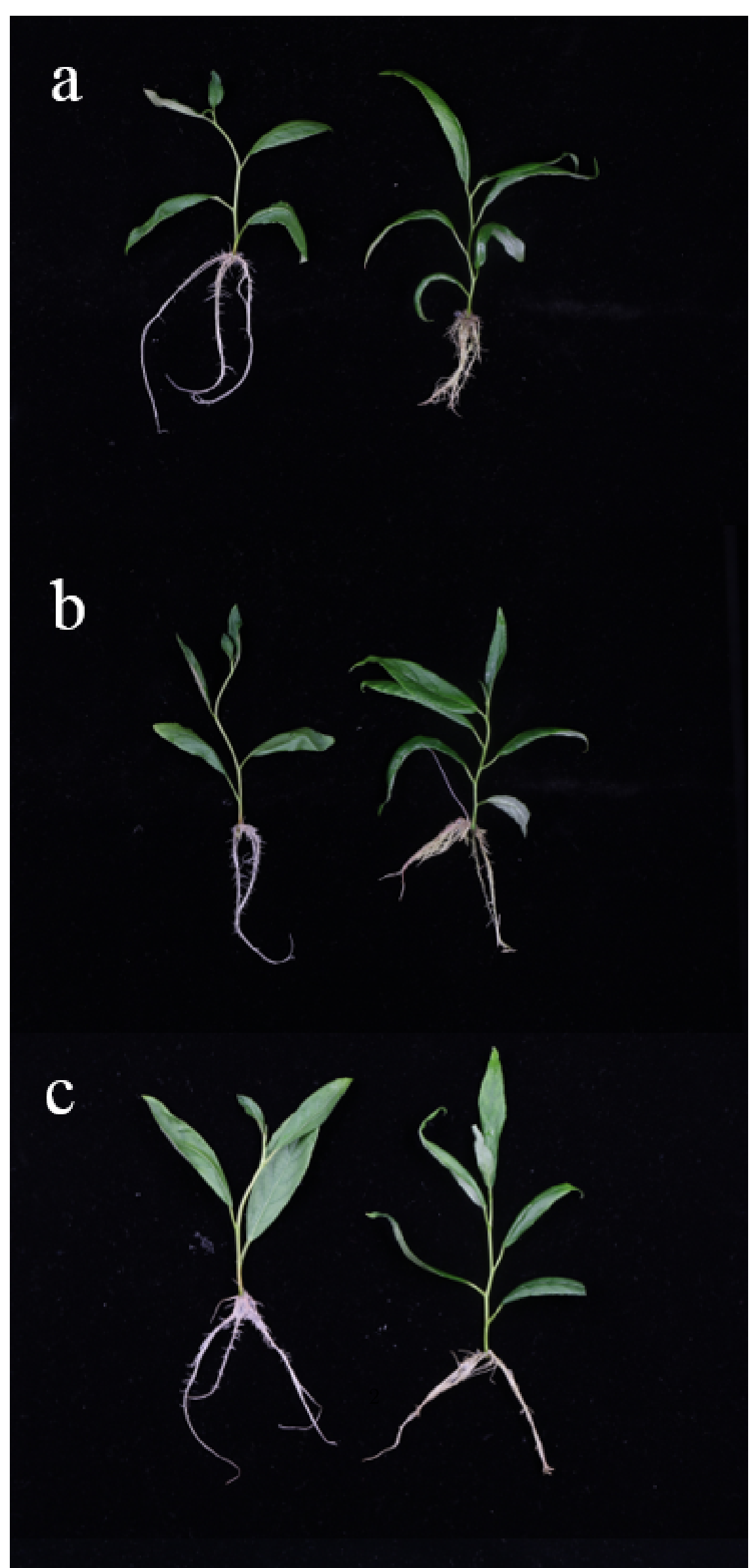


a

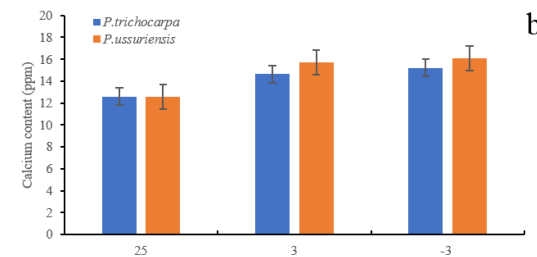

c

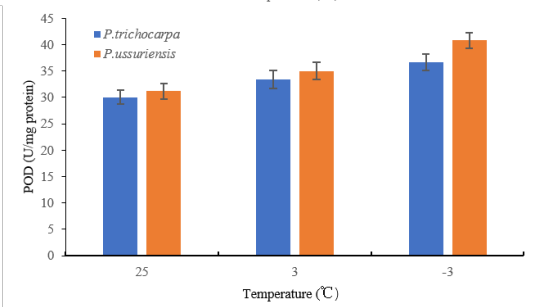

b
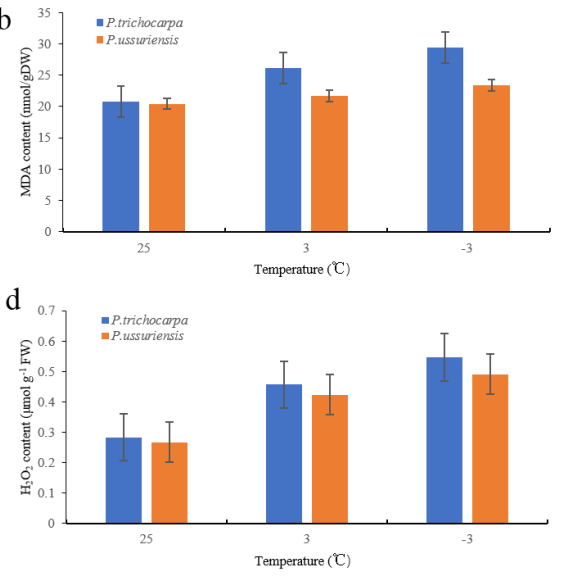

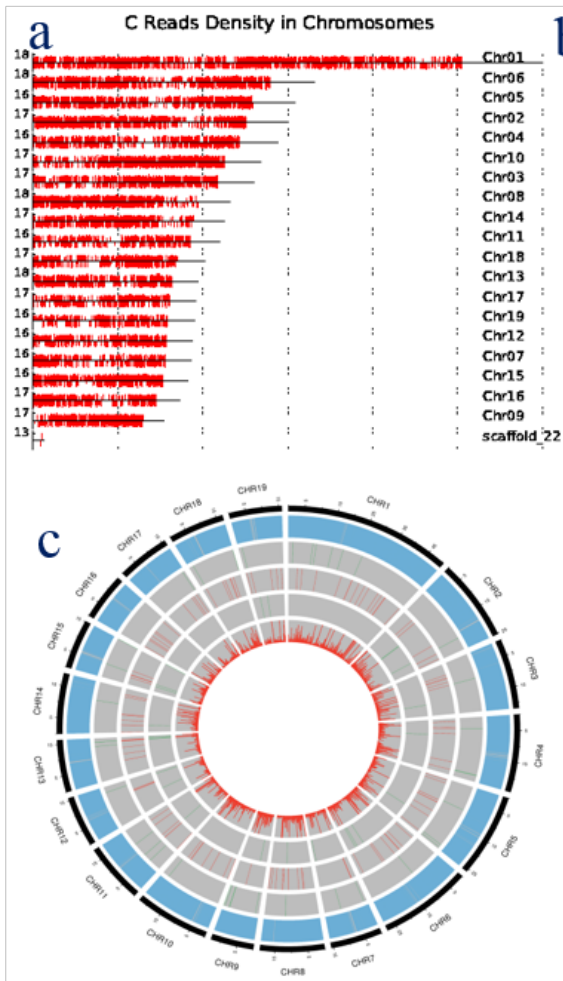

P Reads Density in Chromosomes

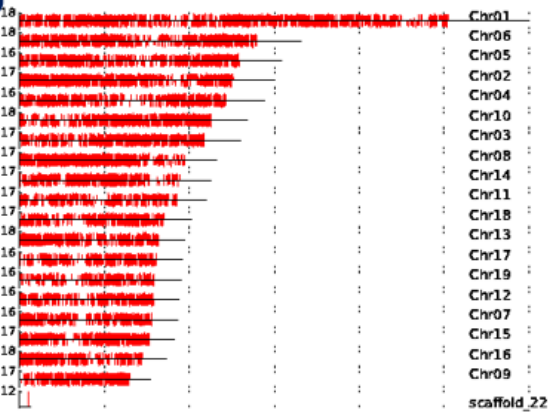

${ }^{12}$

scaffold 22

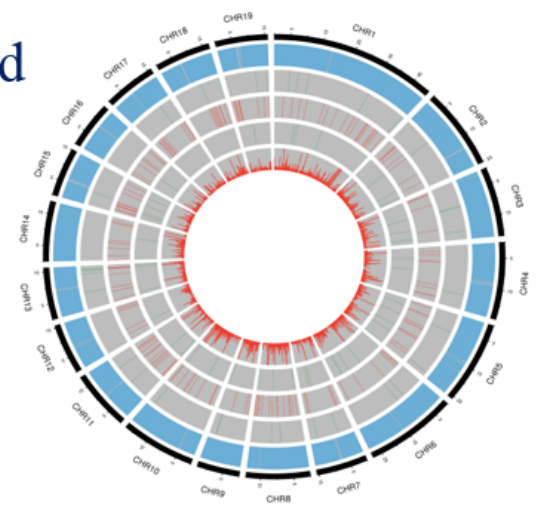




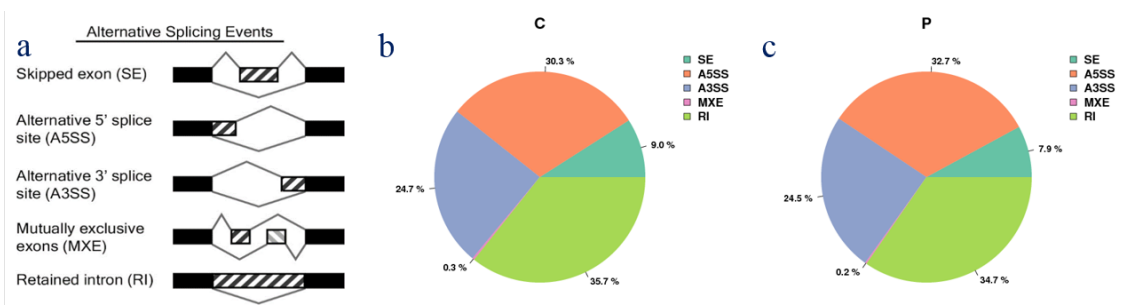

$\square$ Consitutuive exon
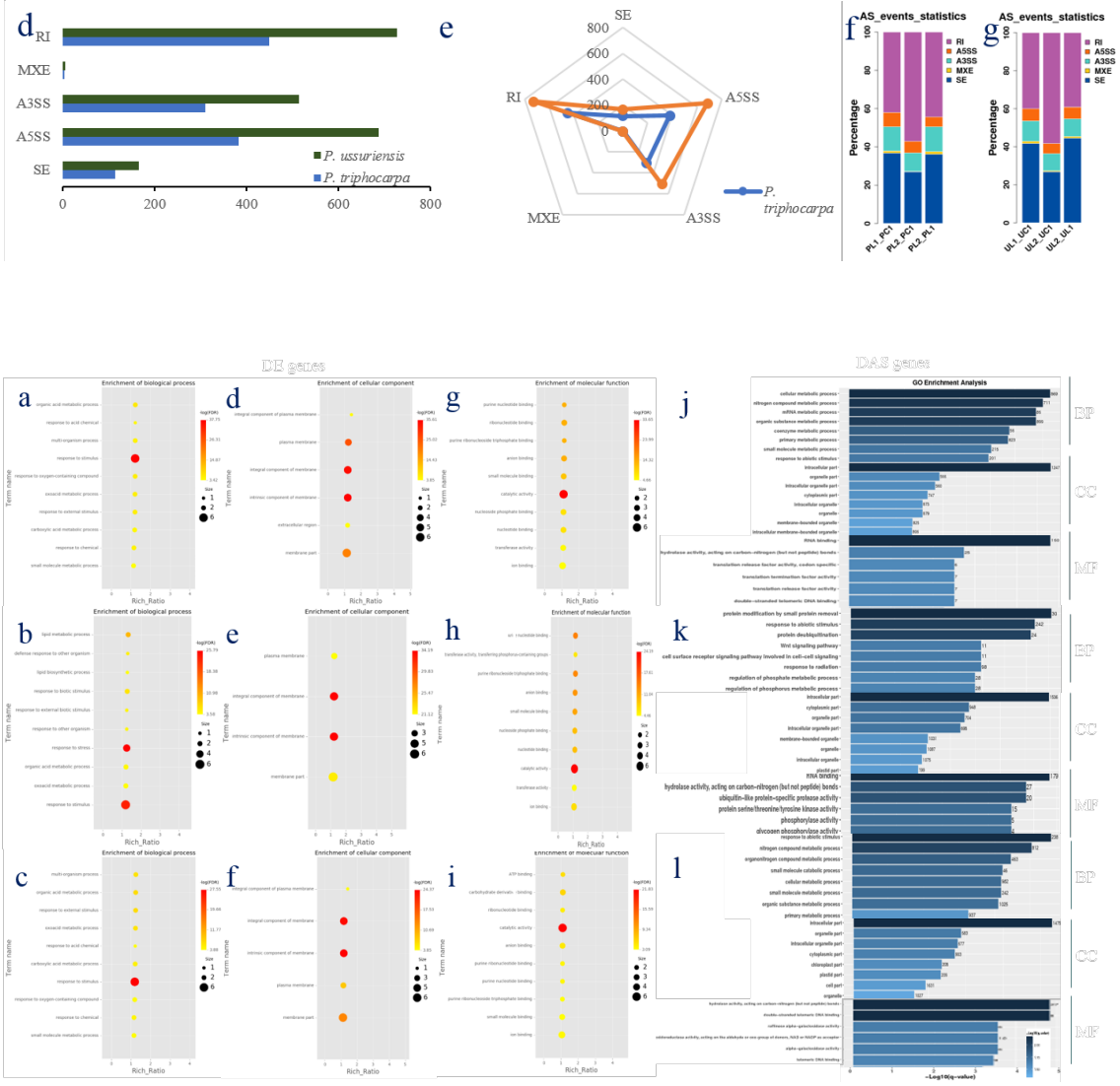

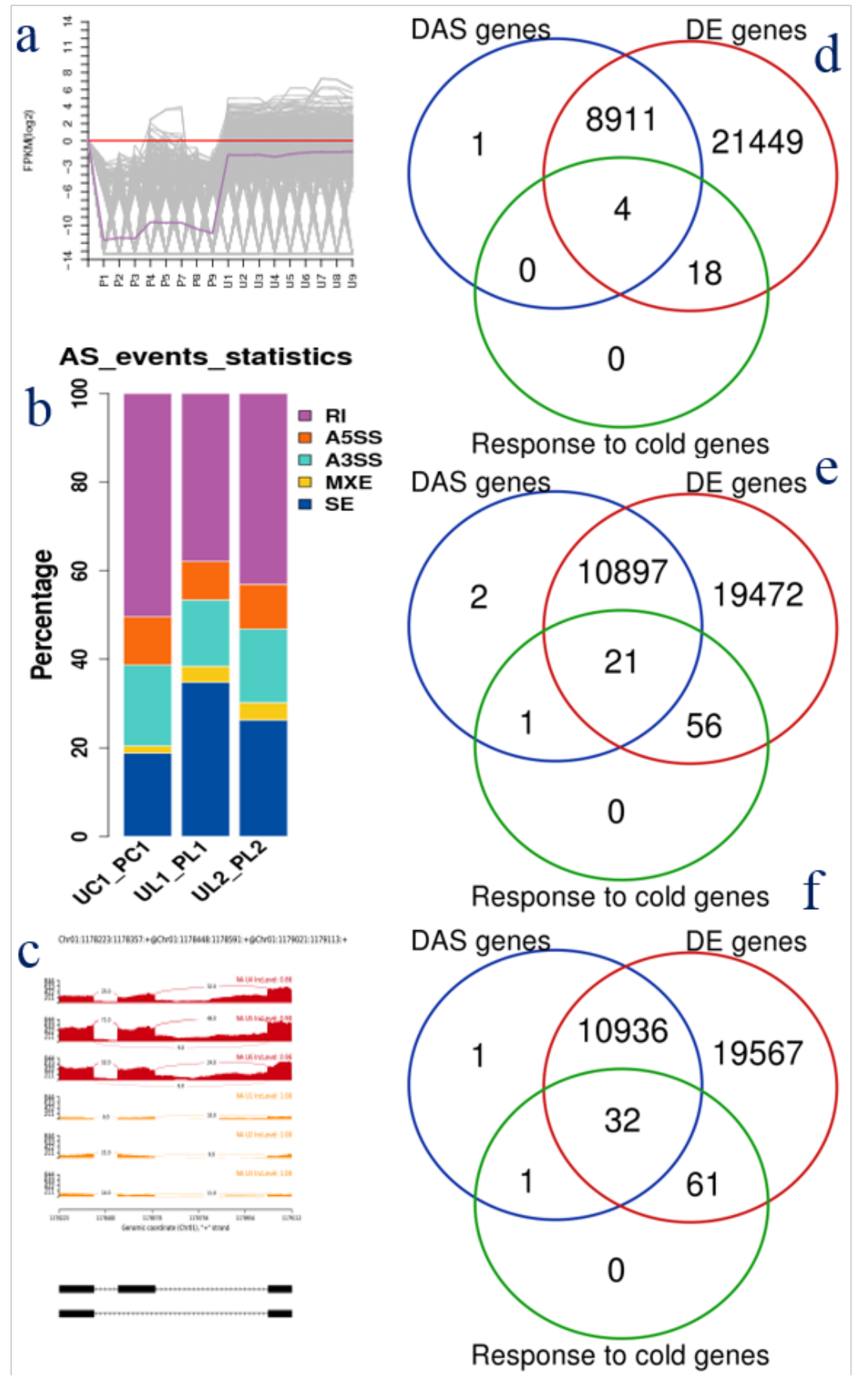

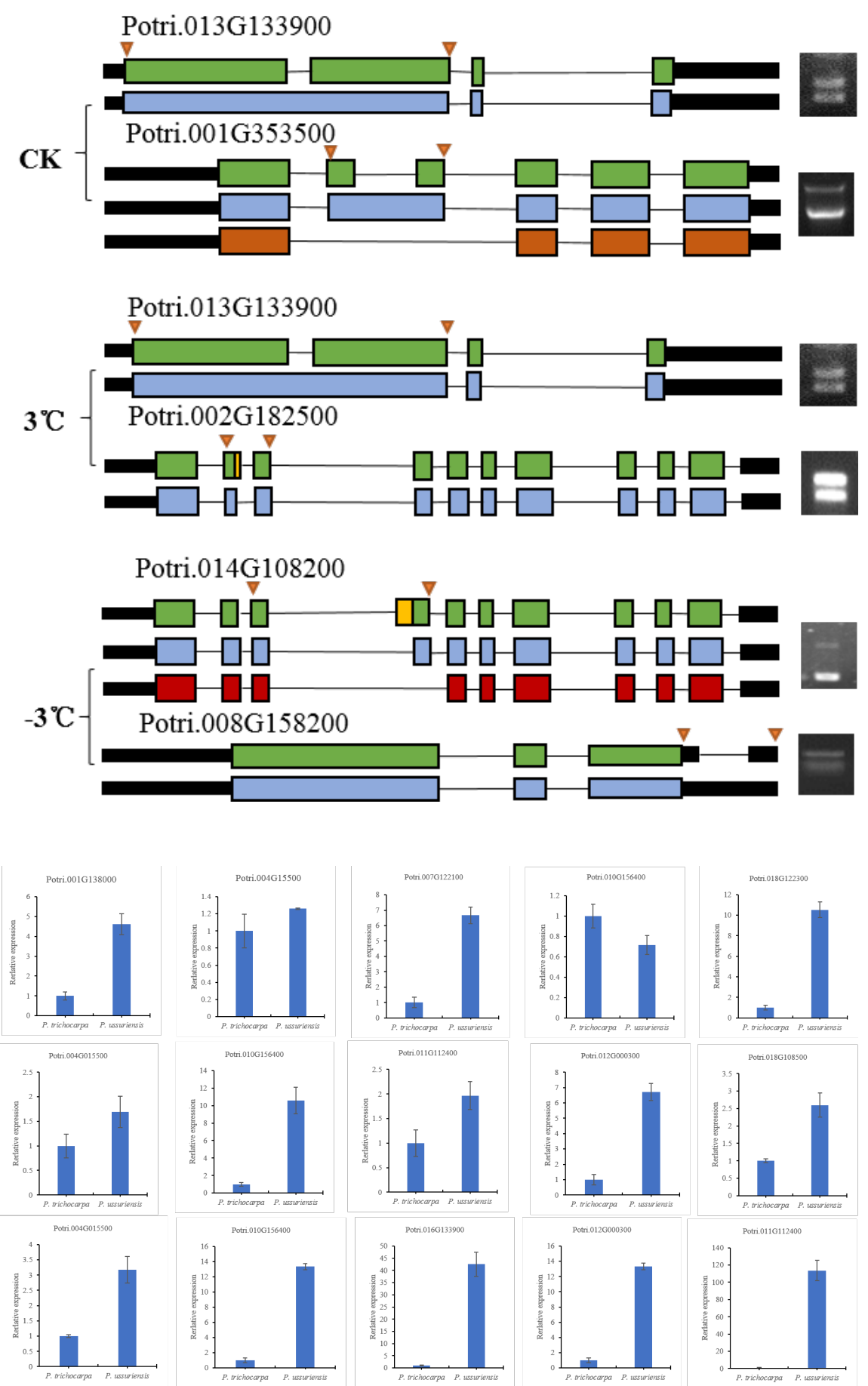


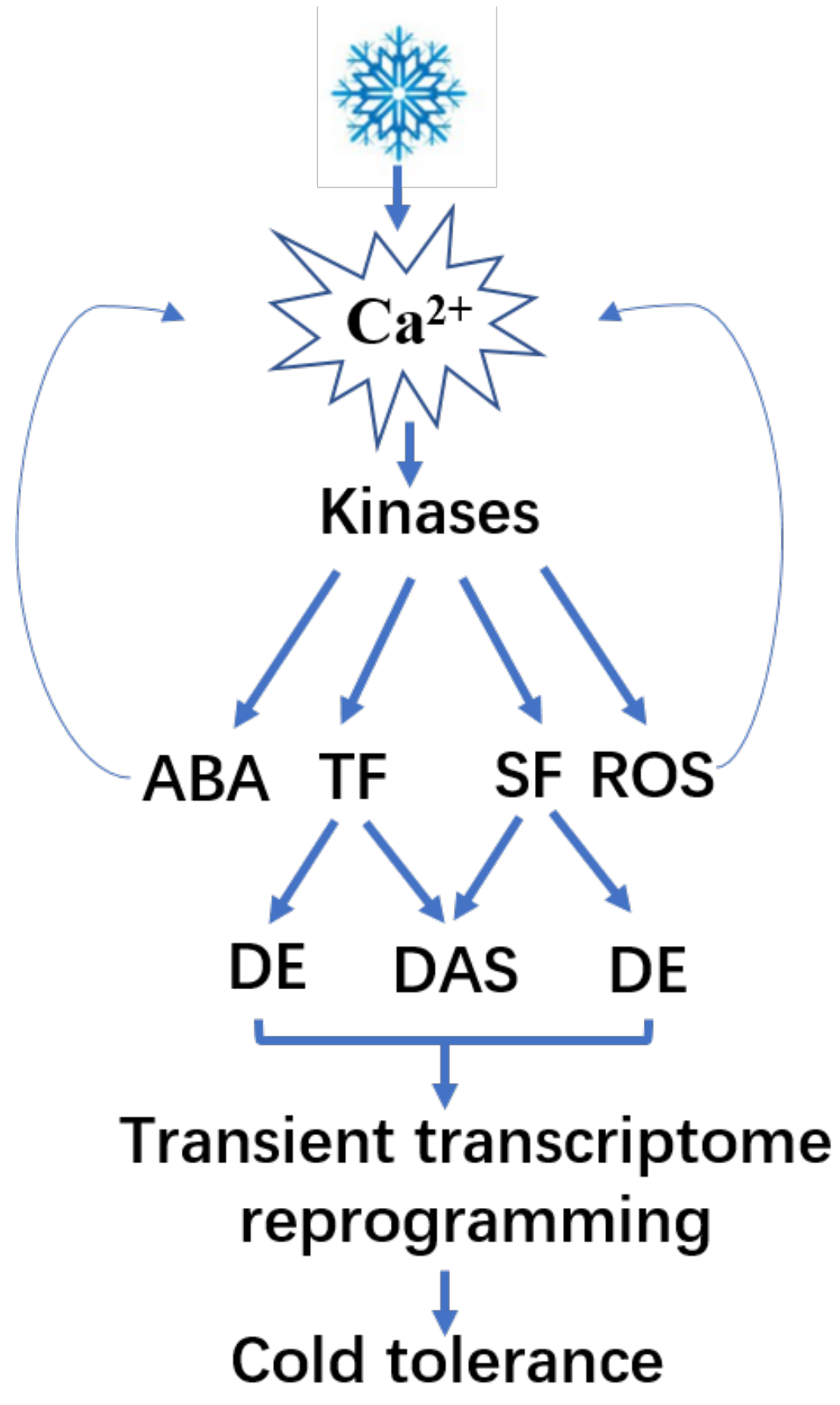

